Boletín de la Sociedad Zoológica del Uruguay, 2021

Vol. 30 (1): $70-83$

ISSN 2393-6940

https://journal.szu.org.uy

DOI: https://doi.org/10.26462/30.1.8

\title{
ENSAYO
}

\section{GYMNOTUS OMARORUM COMO MODELO PARA ESTUDIAR LAS BASES NEUROENDÓCRINAS DE LA AGRESIÓN TERRITORIAL NO REPRODUCTIVA}

\author{
Lucía Zubizarreta ${ }^{1,2}$
}

1. Laboratorio de Neurofisiología Celular y Sináptica, Departamento de Fisiología, Facultad de Medicina, Universidad de la República, Avenida Gral. Flores 2125, Montevideo, Uruguay.

2. Unidad Bases Neurales de la Conducta, Departamento de Neurofisiología Celular y Molecular, Instituto de Investigaciones Biológicas Clemente Estable, Avenida Italia 3318, Montevideo, Uruguay

Izubizarreta@fmed.edu.uy

Fecha de recepción: 31 de agosto de 2020

Fecha de aceptación: 24 de febrero de 2021

\section{RESUMEN}

El estudio de la modulación de la territorialidad en especies silvestres es especialmente adecuado para explorar los mecanismos que subyacen a la plasticidad comportamental, por la cual los animales responden a contextos ambientales y sociales variables. Este ensayo reúne dos marcos conceptuales para explorar las bases neuroendócrinas de la agresión territorial de Gymnotus omarorum, un pez eléctrico nativo que despliega esta conducta durante todo el año, tanto en hembras como en machos. El primer marco conceptual es el ecológico, con la premisa de que al estudiar una especie silvestre es posible analizar en poblaciones naturales los mecanismos proximales resultantes de las presiones evolutivas que moldearon su conducta territorial. El segundo marco conceptual es individual y se centra en el control hormonal esteroideo de la conducta agonística de $\mathrm{G}$. omarorum, debido a que las hormonas esteroides son clave en la integración de información social, ambiental y del estado interno para ejecutar la salida conductual adecuada. Dentro de este marco, la agresión territorial que ocurre disociada del período reproductivo ofrece la oportunidad de estudiar mecanismos de modulación independientes de esteroides gonadales. La conducta agonística territorial no reproductiva de $G$. omarorum ha sido caracterizada en detalle y constituye un comportamiento robusto y complejo, el cual media la adquisición de territorios, tanto en hembras como en machos. En la población estudiada en el ambiente natural, el tamaño de los territorios depende del tamaño corporal, y es independiente del sexo. Los mecanismos hormonales que subyacen a la agresión no reproductiva se exploraron en encuentros diádicos en arenas neutrales en el laboratorio. La conducta agonística no presenta diferencias entre sexos, y la dominancia depende solamente del tamaño corporal. La agresión territorial es independiente de esteroides gonadales y de acciones androgénicas rápidas. Sin embargo, depende de la síntesis rápida de estrógenos, ya que la inhibición de la conversión de andrógenos a estrógenos afectó la ocurrencia de los conflictos, la dinámica, intensidad y resultado de las contiendas. El perfil transcriptómico mostró que el área preóptica de adultos no reproductivos expresa enzimas esteroidogénicas, y luego de la consolidación de la dominancia se evidenció una expresión diferencial de enzimas de las vías esteroideas en dominantes y subordinados. Los individuos no reproductivos presentaron niveles circulantes y cerebrales detectables de andrógenos aromatizables, no mostraron estrógenos circulantes, y presentaron estrona cerebral, lo cual constituye una evidencia de síntesis cerebral de estrógenos. En suma, G. omarorum como único modelo entre los teleósteos, revela que la síntesis cerebral rápida de estrógenos tiene un rol preponderante en la modulación de la agresión no reproductiva, lo que parece ser una estrategia compartida con aves y mamíferos.

\section{ABSTRACT}

Gymnotus omarorum as a model to study the neuroendocrine bases of non-reproductive territorial aggression. The study of the modulation of territoriality in wild species is especially suited to explore the mechanisms underlying behavioral plasticity, by which animals respond to changing environmental and social contexts. This essay brings together two conceptual frameworks to explore the neuroendocrine basis of non-breeding territorial aggression in Gymnotus omarorum, a native electric fish which displays this behavior both in females and males. The first framework 
is ecological, based on the premise that when studying a wild species in its natural settings it is possible to analyze the proximal mechanisms that evolved to shape territorial behavior. The second framework is individual and is focused on the hormonal control of agonistic behavior in $G$. omarorum, because steroids are key in the integration of social, environmental, and internal information to execute an appropriate behavioral output. Within this framework, aggressive behaviors which occurr dissociated from the breeding season encourage the search of non-gonadal underlying regulatory mechanisms. In the natural habitat, territory size is independent of sex and determined by body size. Hormonal mechanisms underlying non-breeding aggression have been explored in dyadic encounters in lab settings, where, in line with what is observed in the natural habitat, males and females display robust aggressive contests, and outcome depends on body size. This agonistic behavior is independent of gonadal hormones and fast acting androgens. Nevertheless, it is dependent on fast acting estrogenic action, as acute inhibition of aromatization affects aggression engagement, intensity, and outcome. Transcriptomic profiling in the preoptic area region shows non-breeding individuals express steroidogenic enzyme transcripts, and after status consolidation dominants and subordinates show differential enzyme expression. Nonbreeding adults display detectable brain and circulating levels of aromatizable androgens, non-detectable levels of circulating estrogens, and high levels of estrone, which constitute a clear evidence of brain synthesis of estrogens. This teleost model reveals there is a role of brain estrogen in the control of non-breeding aggression which seems to be common among distant vertebrate species.

\section{INTRODUCCIÓN}

Los mecanismos que subyacen a la plasticidad comportamental, por la cual los animales responden a contextos sociales y ambientales variables, aún no se comprenden cabalmente. Los comportamientos sociales, aunque complejos, ofrecen la oportunidad de abordar el estudio de la plasticidad comportamental y la riqueza de su modulación. En este sentido, la conducta territorial, como ejemplo de conducta social ampliamente extendida entre los animales, puede aportar información sobre cómo los individuos integran claves externas a su contexto fisiológico para producir respuestas sociales adecuadas. En base a evidencias que sostienen que la conducta agonística media la adquisición y defensa de territorios, este ensayo reúne dos marcos conceptuales para explorar las bases neuroendócrinas de la agresión territorial de Gymnotus omarorum, un pez eléctrico nativo que despliega esta conducta durante todo el año, tanto en hembras como en machos. El primer marco conceptual es el ecológico, con la premisa de que al estudiar una especie silvestre es posible analizar en poblaciones naturales los mecanismos proximales resultantes de las presiones evolutivas que moldearon su conducta territorial. El segundo marco conceptual es individual y se centra en el control hormonal esteroideo de la conducta agonística de $G$. omarorum, debido a que las hormonas esteroides son clave en la integración de información social, ambiental y del estado interno para ejecutar la salida conductual adecuada. Dentro de este marco, es mucho lo que se sabe de la modulación hormonal androgénica de la agresión reproductiva entre machos, mientras que la agresión no reproductiva está mucho menos explorada. Aunar estos dos marcos conceptuales en un solo estudio es de por sí novedoso, y solo posible por las características únicas del modelo utilizado. Intentar que dialoguen y se complementen en sus hallazgos es aún más ambicioso y es uno de los principales propósitos de este trabajo.

\section{TERRITORIALIDAD Y CONDUCTA AGONÍSTICA EN EL MARCO DE LA ECOLOGÍA DEL COMPORTAMIENTO}

\subsection{Espacialidad}

La distribución espacial de los animales en la naturaleza resulta de la interacción de los individuos con el ambiente y está fuertemente influenciada por la presencia o ausencia de alimento, predadores y coespecímenes. En una población, los individuos pueden estar agregados espacialmente o mostrar un patrón disperso (Brown y Orians, 1970). Un patrón disperso no uniforme (irregular) puede deberse a la heterogeneidad en las variables ambientales o distribución de recursos relevantes para los individuos (Eason, Cobbs y Trinca, 1999; Thomas y Kunin, 1999; Moorcroft, Lewis y Crabtree, 2006). Asimismo, una distribución irregular puede explicarse por la existencia de conducta territorial (Maher y Lott, 2000; Morales et al., 2010). La territorialidad garantiza el acceso exclusivo a un determinado recurso, lo cual ofrece una ventaja competitiva y puede redundar en un aumento de la eficacia reproductiva (Davies y Hartley, 1996; Grant, 1997; Rubenstein y Alcock, 2018).

Está ampliamente documentado en muchos vertebrados que durante el período reproductivo los machos defienden territorios y evitan la intrusión de otros machos mediante despliegues de advertencia, amenaza o agresión física (Brown, 1964; Clarke, 1970; Armitage, 1977; Davies, 1976; Heike Pröhl, 2005; Huang, Greene, Chang y Shine, 2011). Aunque es menos frecuente, en algunas especies la defensa territorial puede darse durante todo el año tanto en machos como en hembras (Caldwell, Glickman y Smith,1984; Wingfield y Hahn, 1994; Chiver, Stutchbury y Morton, 2014;). En estos casos la defensa de un territorio puede asegurar acceso a áreas de forrajeo durante todo el año (Davies, Krebs y West, 2012). 


\subsection{Conducta agonística}

La conducta agonística es un comportamiento social ampliamente extendido que participa en la resolución de conflictos entre individuos de una misma especie (King 1973; Archer 1988). Los conflictos surgen de la competencia por un recurso limitado como, por ejemplo, espacio, alimento, pareja o sitios de apareamiento, cuyo control incrementa el fitness individual (Huntingford y Turner, 1987; King, 1973; Hardy y Briffa, 2013). Las interacciones agonísticas en general siguen las mismas fases estereotipadas de evaluación, contienda y post-resolución, e incluyen 3 tipos principales de comportamientos: despliegues de amenaza, agresión física y despliegues de sumisión (Nelson, 2006; Summers y Winberg, 2006). Durante las interacciones agonísticas diádicas los conflictos se resuelven cuando el individuo dominante obtiene el acceso al recurso en disputa y el subordinado es excluido (Nelson, 2006; Briffa y Sneddon, 2010). Cuando el recurso en disputa es el espacio, el territorio es el área de la cual los intrusos son excluidos mediante despliegues de advertencia, amenaza o ataques (Brown, 1975). La evaluación de factores ambientales y sociales que determinan la calidad de un territorio se integra con los requerimientos individuales que determinan la decisión de si competir o no por un área. Una vez iniciada la contienda, los individuos evalúan continuamente los costos y beneficios de continuar compitiendo o rendirse, para lo cual integran la información de su propia capacidad de lucha (que depende del tamaño corporal y armamento, entre otras cosas), con el valor del recurso, que es variable entre individuos dependiendo de su estado reproductivo, sexo, residencia, o disponibilidad de recursos. Los factores que influencian la capacidad de lucha y la estimación del valor del recurso generalmente presentan diferencias sexuales. Incluso en especies con monomorfismo en la capacidad de lucha (por ejemplo, por no presentar diferencias en el tamaño corporal entre sexos) muchas veces se evidencia que el resultado de las contiendas es sesgado hacia uno de los sexos por valorar el recurso de manera asimétrica (por ejemplo, en lémures donde las hembras tienen más probabilidad de ser dominantes, Dunham, 2008).

La diversidad y complejidad de los despliegues agonísticos en vertebrados han generado dificultades para establecer patrones claros que unifiquen los mecanismos y causas evolutivas subyacentes (Goodson y Kabelik 2009). Por esta razón, el uso de modelos animales no tradicionales y la exploración multidisciplinaria de su comportamiento marcan el rumbo de las investigaciones actuales que intentan comprender las bases neuroendócrinas de esta conducta tan extendida en vertebrados (Blumstein et al. 2010). La agresión en machos es un comportamiento más conspicuo que en hembras, si bien en los dos sexos la interpretación desde el punto de vista de la selección natural constituye un aumento de la eficacia reproductiva (Bateman 1948; Trivers
1972; Emlen y Oring 1977; Shuster y Wade, 2003). La agresión entre hembras es muy extendida, y se ha demostrado que ocurre en contextos de competencia por rango social, territorios y acceso a pareja, sin embargo, ha sido relegada de las investigaciones y se conoce menos sobre sus mecanismos proximales de control(Rosvall, 2011; Cain y Rosvall, 2014).

\subsection{Determinantes del territorio}

El estudio de la modulación de la conducta territorial en especies silvestres es especialmente adecuado para explorar los mecanismos que subyacen a la plasticidad comportamental. En la conducta territorial, los animales primero detectan las claves ambientales y sociales que determinan el valor del territorio, luego integran esta información con sus requerimientos individuales y capacidad de lucha, y finalmente evalúan si competir por un área o no. Por lo tanto, la distribución espacial de individuos de una especie territorial aporta información sobre cómo los animales integran sus características individuales con factores ambientales y sociales. La variación en la habilidad para la obtención y defensa de un territorio puede generar diferencias en el tamaño del mismo, ya que características como el tamaño corporal, el sexo, y el estado reproductivo determinan la capacidad de lucha e influyen sobre la estimación del valor del recurso (Smith y Parker, 1976; Hurd, 2006; Piper et al., 2015; Metcalfe, Van Leeuwen, y Killen, 2016; Nolen, Allen y Miller, 2017). El tamaño corporal se asocia con el área del territorio en muchas especies, posiblemente por determinar directamente los requerimientos metabólicos, y por influenciar el resultado de las contiendas agonísticas -(Elliott, 1990; Butchart, Seddon y Ekstrom, 1999; Keeley, 2000; Adams, 2001). En especies que despliegan defensa territorial en ambos sexos, las asimetrías en la capacidad de lucha y los factores motivacionales pueden provocar diferencias sexuales en el tamaño del territorio. Por ejemplo, en ardillas (Sciurus vulgaris), los machos poseen territorios de mayor tamaño que las hembras, a pesar de presentar monomorfismo en el tamaño corporal - (Wauters y Dhondt, 1992). En lagartijas (Sceloporus virgatus), las hembras despliegan mayor conducta territorial que los machos debido a razones motivacionales (Smith, 1985).

\section{BASES NEUROENDÓCRINAS DE LA TERRITORIALIDAD Y LA CONDUCTA AGONÍSTICA}

\subsection{Hormonas esteroides}

Las hormonas esteroides son actores fundamentales en la regulación de todos los comportamientos sociales, incluida la agresión y coordinan respuestas conductuales integradas a claves fisiológicas, sociales y ambientales (McEwen y 
Wingfield, 2003; Hirschenhauser y Oliveira, 2006). En vertebrados las hormonas esteroides se pueden clasificar según la fuente de producción en: aesteroides sexuales, producidos principalmente por los ovarios y testículos (andrógenos, estrógenos y progestágenos); y b- corticosteroides, producidos principalmente por la corteza adrenal o tejido interrenal (en peces y anfibios). Independientemente de la clasificación referida a la fuente, los esteroides pueden producirse en células localizadas fuera de las gónadas y adrenales, como el tejido adiposo y el cerebro. Algunos esteroides presentan variaciones entre especies; por ejemplo, en peces y primates el principal corticosteroide es el cortisol, mientras que en roedores es la corticosterona (Adkins-Regan, 2005). Otro ejemplo puede ser el caso de la 11-Ketotestosterona (11-KT), un andrógeno presente en grandes concentraciones en peces teleósteos, y ausente en otros taxa (Borg, 1994). Lo que determina las diferencias de producción entre tejidos y diferencias interespecíficas es la variación en la expresión de enzimas esteroidogénicas. La secreción y concentración de los esteroides se regula principalmente por la tasa de síntesis, y metabolismo, ya que el almacenamiento es casi nulo.

Los esteroides modulan la conducta a través de dos mecanismos de acción. El primero es mediado por receptores intracelulares que actúan como factores de transcripción (Harding, 1992; Fowler, Freeman y Wang, 2003; Micevych, Hammer y Hammer, 2005). En este modo de acción los esteroides actúan como factores permisivos, pero no son por lo general el gatillo de la conducta, y deben estar presentes en altas concentraciones y de manera sostenida. Las hormonas esteroides pueden cambiar los umbrales para que otros factores modulen la salida comportamental, y así afectar, por ejemplo, el umbral de respuesta frente al estímulo de un co-especimen. EI concepto de las hormonas como factores permisivos de una conducta dependiendo del contexto, se vuelve especialmente importante en la consideración de las conductas sociales. A pesar de que el rol de las hormonas esteroides como factores permisivos es el más estudiado, estudios recientes han demostrado que existe síntesis local de hormonas esteroides en el cerebro y se ha descubierto que también ejercen acciones rápidas mediadas por receptores de membrana, similares a las de los neuromoduladores (Remage-Healey, 2014).

\subsection{Modulación hormonal de la agresión territorial}

A pesar de que los despliegues de los comportamientos agonísticos presentan un alto grado de variabilidad, los circuitos neurales subyacentes a estas conductas, los ejes endócrinos que los regulan (ej.: hipotálamo-hipófisis-gonadal) y las hormonas involucradas (ej.: andrógenos, estrógenos, neuropéptidos hipotalámicos) se encuentran altamente conservados en vertebrados (Goodson,
2005). Las áreas del cerebro asociadas al control de los comportamientos sociales son un conjunto de núcleos interconectados conocido como Red del Comportamiento Social (RCS; Goodson y Kabelik, 2009; O' Connell y Hofmann, 2011; O Connell y Hofmann, 2012). Una característica común a los nodos de la RCS es poseer receptores de hormonas esteroides y neuropéptidos hipotalámicos, lo que señala la relevancia de estas hormonas en la regulación de la actividad de esta red (Goodson y Kabelik, 2009). Se postula que el patrón de activación espacio temporal de los núcleos que componen la RCS difiere según la situación conductual en la que se encuentra el animal. De esta manera, por ejemplo, los circuitos neurales que gobiernan conductas tan diferentes como la agresión y la reproducción poseen elementos neurales compartidos, aunque activados en forma diferente dependiendo del contexto.

Está bien demostrado que las hormonas esteroides (andrógenos y estrógenos) contribuyen a la expresión de la conducta agonística modulando el patrón de activación de la RCS, aunque los mecanismos precisos por los que actúan son aún objeto de investigación. A mediados del siglo XIX los experimentos pioneros de Berthold (revisado en Soma, 2006) establecieron que un factor testicular (luego descripto como testosterona) promovía las características sexuales secundarias y la agresión en gallos, sentando las bases de la endocrinología conductual. El estudio de la regulación endócrina de la agresión territorial ha sido mayormente abordado en machos reproductivos, en los cuales se ha establecido como principio general que los niveles elevados de andrógenos gonadales promueven la agresión (Fig. 1A; revisado en Cunningham, Lumia y McGinnis, 2012; Fuxjager, Trainor y Marler, 2017). Aunque la testosterona es una hormona fundamental en la regulación de la agresión en machos, muchos estudios que hilaron más fino demostraron que la relación entre los andrógenos circulantes y la agresión es más compleja. Otros factores como la expresión diferencial de receptores, síntesis cerebral de esteroides, o el contexto social juegan un papel fundamental en los efectos de los andrógenos circulantes en la agresión. En 1990 se demostró por primera vez que la aromatización de andrógenos a estrógenos es necesaria para el despliegue de agresión en machos de codorniz Coturnix coturnix japonica (Schlinger y Callard, 1990). Luego se mostró en otras especies que las acciones androgénicas sobre la agresión en machos están mediadas en parte por la aromatización de andrógenos a estrógenos (Fig. 1B; revisado en Trainor, Kyomen y Marler, 2006). Más adelante, dos modelos alternativos ofrecieron nuevas miradas sobre la regulación hormonal de la agresión: la agresión en hembras (Rubenstein y Wikelski, 2005; Renn, Fraser, Aubin-Horth, Trainor y Hofmann, 2012; Rosvall et al., 2012; Scaia et al., 2018), revisado en 
A

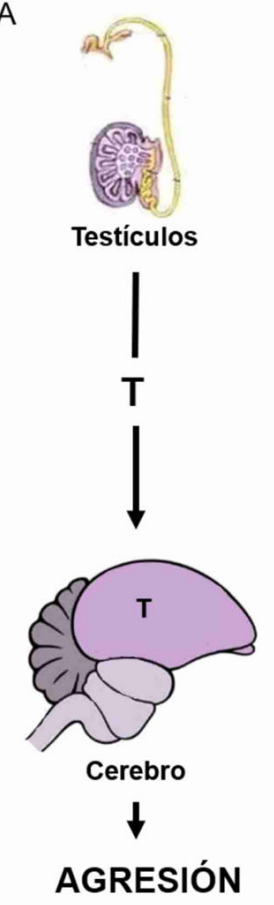

B

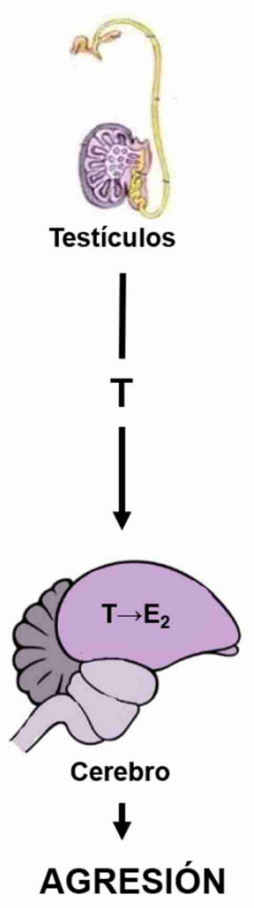

C

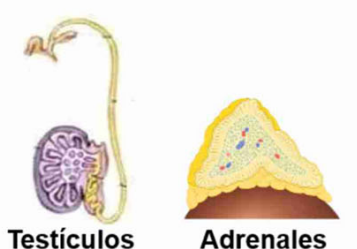

Testiculos

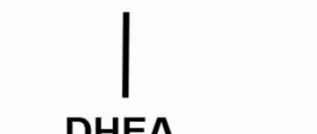

DHEA

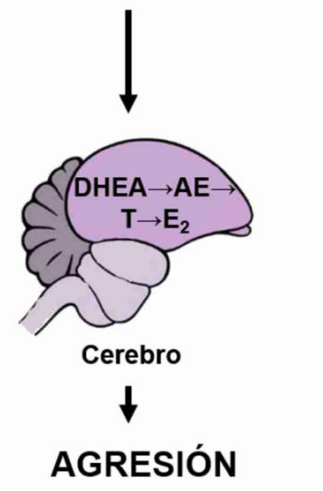

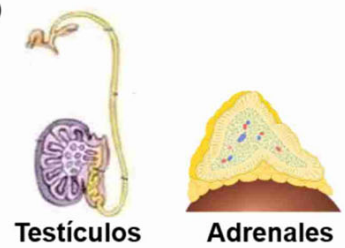

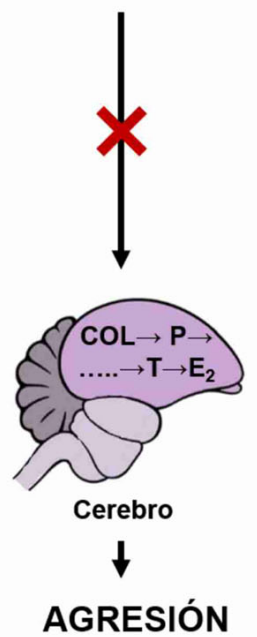

Fig. 1. Vías por las cuales los esteroides sexuales pueden modular la conducta agonística en machos. A- La testosterona gonadal actúa directamente en el cerebro. B- La testosterona gonadal se aromatiza a $17 \beta$-estradiol en el cerebro. C- La DHEA adrenal se convierte en testosterona y $17 \beta$-estradiol en el cerebro. D- La testosterona y el $17 \beta$-estradiol se producen de novo en el cerebro a partir del colesterol.

El cambio de una señalización circulante (A y B), a una producción cerebral local (C y D) usualmente se acompaña de un cambio en los mecanismos de acción esteroideos; es decir, de acciones lentas genómicas en Ay B, a acciones rápidas no genómicas en $\mathrm{C}$ yD. Modificado de (Jalabert etal., 2018)

$\mathrm{CHOL}=$ colesterol; $\mathrm{PREG}=$ pregnenolona; $\mathrm{DHEA}=$ dehidroepiandrosterona; $\mathrm{AE}=$ =androstenediona; $\mathrm{T}=$ testosterona; $\mathrm{E} 2=17 \beta-$ estradiol.

(Rosvall, 2011; Duque-Wilckens y Trainor, 2017; Rosvall, Bentz y George, 2019); y la agresión fuera del período reproductivo (revisado en Soma et al., 2008, 2015).

La agresión en hembras es prevalente en muchas especies y adaptativa en varios contextos. Además de la bien documentada agresión maternal (Mayer y Rosenblatt, 1987; Smiley, Ladyman, Gustafson, Grattan y Brown, 2019), las hembras pueden competir por rango social, territorios o acceso a pareja (revisado en Rosvall, 2013). En particular, la agresión territorial en hembras ha sido reportada en peces, reptiles, aves y mamíferos (revisado en Duque-Wilckens y Trainor, 2017). Tanto la testosterona como los estrógenos pueden incrementar la agresión en hembras (Woodley y Moore, 1999; Rubenstein y Wikelski, 2005; Scaia et al., 2018), revisado en (Rosvall et al., 2019), aunque el efecto de los estrógenos sobre la agresión puede variar, dependiendo del subtipo de receptor involucrado (Clipperton-Allen, Cragg, Wood, Pfaff y Choleris, 2010; Clipperton-Allen, Almey, Melichercik, Allen y choleris, 2011).. La agresión territorial fuera del período reproductivo ha sido reportada en diversas especies y constituye un paradigma ideal para estudiar la neuromodulación de la agresión con independencia del factor gonadal (Logan y Wingfield 1990; Jasnow, Huhman, Bartness y Demas, 2000; Hau, Stoddard y Soma, 2004). La agresión territorial en este período (en el que las gónadas regresadas sintetizan niveles muy bajos de esteroides) es independiente de la testosterona circulante (Soma et al., 2008; Logan y Wingfield, 1990; Hau et al. 2004). De hecho, la castración no disminuye la conducta agresiva territorial en varias especies (Wingfield, 1994; Pinxten et al. 
2000). Se ha observado incluso que los machos de estas especies no aumentan los niveles de testosterona en interacciones agresivas cuando se le presenta un intruso dentro de su territorio fuera del período reproductivo (Wingfield y Monk 1994). El mantenimiento de la agresividad en el período no reproductivo, con bajos niveles plasmáticos de esteroides sexuales, puede ser explicado por al menos dos mecanismos que involucran igualmente a las hormonas esteroides (Wingfield et al., 2001).

a.- Precursores inertes de esteroides son sintetizados en los órganos periféricos y convertidos a hormonas esteroides bioactivas en el cerebro. En aves se encontraron concentraciones plasmáticas elevadas de dehidroepiandrosterona (DHEA, un precursor adrenal de hormonas esteroides) y la capacidad de metabolizarlo a nivel central (Fig. 1C). El tratamiento con DHEA aumenta la agresividad (Soma et al., 2008) y sus niveles plasmáticos correlacionan con la agresividad en machos(Hau et al., 2004).

b.- Síntesis de novo de esteroides sexuales en el cerebro a partir del colesterol, independientemente de las gónadas y otros tejidos periféricos (Fig. 1D). Este proceso fue reportado en mamíferos (Tsutsui et al., 2000) y aves (Pradhan et al., 2010; Soma et al., 2008).

Los estrógenos sintetizados en el cerebro tienen un rol preponderante en la regulación de la agresión no reproductiva en machos, actuando mayormente a través de mecanismos rápidos (Demas et al., 2007; Soma et al., 2000; Tsutsui y Yamazaki, 1995). En machos de aves y mamíferos que despliegan agresión no reproductiva, el tratamiento con 17- $\beta$ Estradiol promueve rápidamente la agresión (Trainor, Lin, Finy, Rowland y Nelson, 2007; Trainor, Sima Finy y Nelson, 2008; Laredo et al., 2013; Heimovics, Trainor y Soma, 2015; Merritt et al., 2018), revisado en (Heimovics et al., 2015), mientras que la inhibición aguda de la aromatasa (enzima que transforma andrógenos en estrógenos) disminuye los niveles de agresión no reproductiva en machos de aves y peces (Soma et al., 2000; Jalabert, Quintana, Pessina y Silva, 2015) . La regulación de la agresión no reproductiva en hembras ha sido abordada en pocas especies. En modelos de aves hay evidencia que apoya el involucramiento de la DHEA y testosterona circulantes (Hau et al., 2004; Gill, Alfson y Hau, 2007), mientras que en el hámster dorado la agresión no reproductiva es independiente de las hormonas ováricas (Fleming, Phillips, Rydall y Levesque, 1988). En el hámster siberiano, las hembras no reproductivas, a pesar de exhibir bajos niveles de hormonas gonadales, despliegan agresión de manera robusta asociada a un aumento de la sensibilidad cerebral al 17- $\beta$ Estradiol (Scotti, Place y Demas, 2007; Rendon, Amez, Proffitt, Bauserman y Demas, 2017). Por lo tanto, mientras que los efectos rápidos de los estrógenos han sido documentados en la agresión no reproductiva de machos, actualmente no está claro si este tipo de modulación ocurre en hembras no reproductivas.

\section{GYMNOTUS OMARORUM COMO MODELO PARA ESTUDIAR LAS BASES NEUROENDÓCRINAS DE LA AGRESIÓN}

La agresión territorial disociada del período reproductivo se ha reportado en diversos taxa, incluyendo aves (Logan y Wingfield, 1990; Gwinner, Rödl y Schwabl, 1994; Wingfield y Monk, 1994; Soma, Kiran, Sullivan y Wingfield, 1999), mamíferos (Caldwell et al., 1984; Jasnow et al., 2000; Demas et al., 2007), reptiles (Moore y Marler, 1987), y peces (Batista, Zubizarreta, Perrone y Silva, 2012; Vullioud, Bshary y Ros, 2013). Estas especies ofrecen la oportunidad de estudiar cómo las variables ambientales y características individuales se relacionan con el tamaño territorial, aún en ausencia de hormonas gonadales. El pez eléctrico Gymnotus omarorum despliega el único ejemplo conocido hasta el momento en teleósteos de agresión territorial intrae inter-sexual durante todo el año. Se caracteriza por no presentar dimorfismo sexual morfológico ni electrofisiológico y por ser un reproductor estacional (Richer-de-Forges et al., 2009). Es una especie autóctona abundante, por lo que es posible implementar estudios de la ecología del comportamiento directamente en poblaciones naturales. Es una especie ampliamente utilizada como modelo neuroetológico, cuyo nombre homenajea a Omar Trujillo-Cenoz y Omar Macadar, los dos pioneros que iniciaron el estudio de los peces eléctricos en Uruguay, quienes lideraron investigaciones sobre la anatomía fisiología y biofísica de la electrogeneración. Al igual que todos los peces eléctricos sudamericanos, G. omarorum emite una descarga eléctrica (DOE) de manera continua, que forma parte de un sistema sensorial activo (electrogeneración/electrorrecepción). Asimismo, modificaciones en la frecuencia y la forma de onda de la DOE constituyen señales de comunicación, utilizadas en los comportamientos sociales.

La agresión territorial no reproductiva de G. omarorum es un comportamiento robusto que se desencadena en una arena neutral por la presencia de un coespecimen tanto en machos como en hembras (Batista et al., 2012). La conducta agonística no reproductiva de $G$. omarorum ha sido descripta en detalle, e incluye un florido repertorio de despliegues locomotores y eléctricos (Fig. 2). Los conflictos presentan una fase de evaluación muy corta, luego de la cual los individuos muestran una escalada agresiva que termina cuando el subordinado deja de atacar y huye (Batista et al., 


\section{CONDUCTA AGONÍSTICA NO REPRODUCTIVA}

\section{Dinámica de la conducta agonística}

$300 \mathrm{~s}$

$400 \mathrm{~s}$

$600 \mathrm{~s}$

\begin{tabular}{c:c|c|} 
evaluación & \multicolumn{1}{|c|}{ contienda } \\
primer ataque & resolución &
\end{tabular}
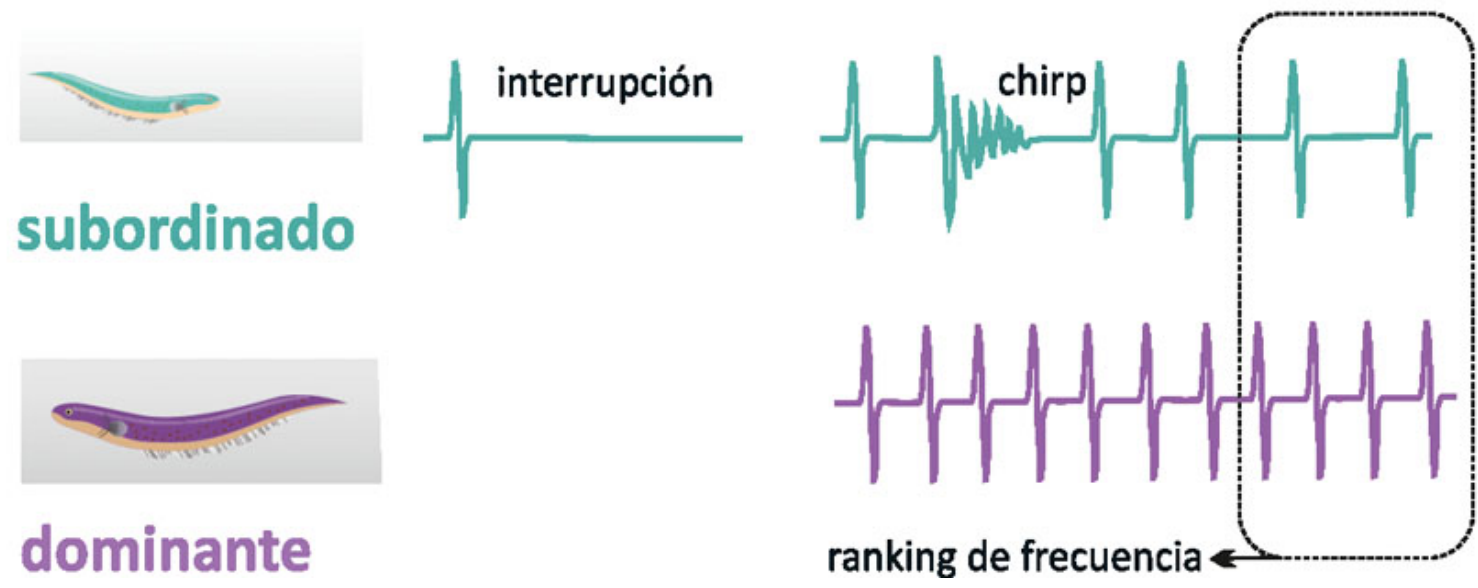

Fig. 2. Conducta agonística no reproductiva en Gymnotus omarorum. Dinámica de los encuentros agonísticos territoriales. Los conflictos presentan tres fases; 1- Evaluación: desde la apertura de la compuerta a la ocurrencia del primer ataque; 2Contienda: desde la ocurrencia del primer ataque hasta la resolución (el subordinado se retira y no vuelve a atacar); C- Postresolución: se analizan 10 minutos luego de la resolución del conflicto. Se ilustran las señales eléctricas de comunicación: Apagado de la descarga del órgano eléctrico (DOE), chirp, y ranking de frecuencia de la DOE (el dominante mantiene una frecuencia mayor que el subordinado luego de la resolución del conflicto).

2012; Zubizarreta, Stoddard y Silva, 2015). Los subordinados señalizan su sumisión eléctricamente utilizando tres tipos de señales de manera secuencial: interrupciones de la DOE, aumentos transitorios de la frecuencia de la DOE con distorsión de la forma de onda (chirps), y por último una disminución sostenida de la frecuencia basal de la DOE luego de la resolución del conflicto (Batista et al., 2012; Silva, Perrone, Zubizarreta, Batista y
Stoddard, 2013; Perrone y Silva, 2018). Una vez que el estatus de dominancia/subordinación se establece, no se revierte y el pez dominante excluye al subordinado del territorio de manera sostenida (Perrone, Pedraja, Valiño, Tassino y Silva, 2019), constituyendo un claro ejemplo en que la conducta agonística media la adquisición y defensa del territorio.

En esta inusual conducta agonística territorial no 


\section{LA CONDUCTA AGONISTICA MEDIA LA TERRITORIALIDAD NO REPRODUCTIVA}

\section{En el laboratorio (corto plazo)}

OCURRENCIA DE CONFLICTO

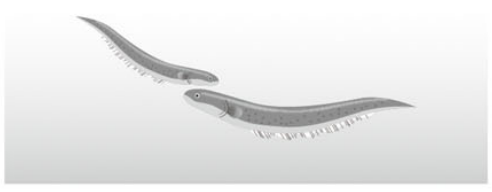

Independiente de - sexo

\section{ESTATUS Y TERRITORIALIDAD}

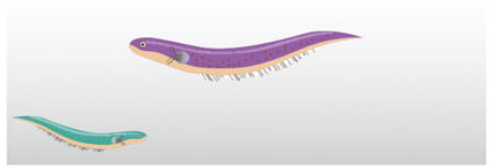

- hormonas gonadales

- acción androgénica rápida

Dependiente de - tamaño corporal - acción estrogénica rápida

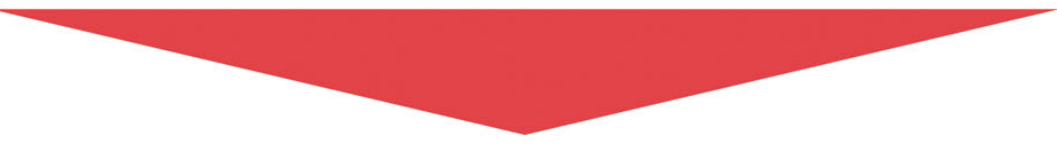

\section{En el hábitat natural (largo plazo)}

\section{TAMAÑO DE TERRITORIO}

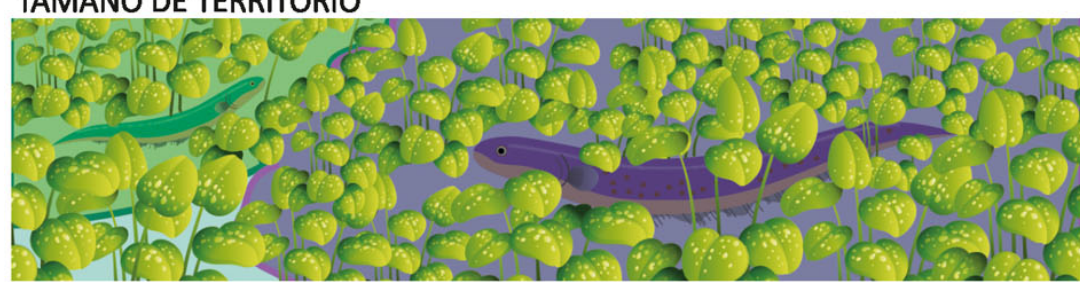

Independiente de - sexo

Dependiente de - tamaño corporal

Fig. 3. Gymnotus omarorum como modelo para estudiar las bases neuroendócrinas de la agresión territorial no reproductiva. Se resumen evidencias provenientes de estudios en el ambiente natural y el laboratorio. Modificado y actualizado de (Silva etal., 2020). reproductiva, el resultado de una contienda depende del tamaño corporal, y el sexo no impacta en la probabilidad de ser dominante (Batista et al., 2012). Más aún, la conducta agonística no presenta sesgo por sexo en ninguno de sus parámetros (Batista et al., 2012; Quintana et al., 2016). Las características de la conducta estudiada en el laboratorio se ven reflejadas en la distribución espacial de los individuos en la naturaleza. Durante el período no reproductivo los adultos ocupan sitios individuales separados por una distancia promedio de aproximadamente 1 metro. El sexo de los individuos no impacta en la distribución espacial, dado que el sexo del vecino más cercano es indistinto. El tamaño del territorio correlaciona positivamente con el tamaño corporal, y no presenta sesgo por sexo Zubizarreta, Silva y Quintana, 2020a).

La agresión territorial no reproductiva de $G$. omarorum es independiente de hormonas gonadales. Un desafío territorial no modifica los niveles de 11-KT circulantes en machos, y la gonadectomía no afecta la conducta agonística no 
reproductiva (Jalabert et al., 2015; Quintana et al., 2016). Esto demuestra que los bajos niveles de esteroides gonadales circulantes que presentan estos animales no reproductivos no son necesarios para la ocurrencia de esta conducta. El rol de los esteroides extra-gonadales se puso a prueba a través de manipulaciones farmacológicas, donde se evaluó el efecto de antagonizar a corto plazo las vías estrogénica y androgénica. La inhibición en agudo de la síntesis de estrógenos provoca una distorsión de la dinámica de las contiendas, una disminución de los niveles de agresión/sumisión y una dificultad para la resolución del conflicto (Jalabert et al. 2015; Zubizarreta et al,. 2020a). El efecto directo de los andrógenos se descartó mediante la administración en agudo de un bloqueante de los receptores de andrógeno, que no provoca cambios en la agresión no reproductiva (Zubizarreta et al. 2020b). En suma, la aromatización de andrógenos a estrógenos, que claramente no ocurre en las gónadas, es fundamental para el mantenimiento de la conducta agonística territorial no reproductiva de G. omarorum.

El postulado que se impone para explicar la modulación esteroidea de la agresión territorial no reproductiva en $\mathrm{G}$. omarorum es que sea mantenida por síntesis cerebral de estrógenos. Este postulado se sostiene también por dos conjuntos de evidencias complementarias que provienen de un abordaje transcriptómico y de cuantificación de esteroides circulantes y cerebrales en individuos no reproductivos. El cerebro de individuos no reproductivos de $G$. omarorum expresa enzimas de las vías esteroidogénicas (Eastman et al., 2020). La defensa territorial y el mantenimiento del estatus de dominancia/subordinación desencadenan cambios genómicos relacionados a las vías esteroideas. En dominantes se constató un aumento en la transcripción de aromatasa, mientras que en subordinados se produjo un aumento en la expresión de enzimas que metabolizan estrógenos y andrógenos (Eastman et al., 2020). Por otro lado, en individuos no reproductivos se detectó la presencia de andrógenos aromatizables circulantes y cerebrales (androstenediona y testosterona), así como la ausencia de estrógenos circulantes, y neurosíntesis de estrógenos(Zubizarreta, 2020).

La contribución de Gymnotus omarorum, un teleósteo con agresión persistente desacoplada de la reproducción estacional, expande los conceptos basados en aves y mamíferos a un representante de un grupo troncal de los vertebrados, para entender la modulación hormonal de la agresión no reproductiva. La principal consecuencia de integrar los marcos ecológico y neuroendócrino es la posibilidad de identificar la secuencia de eventos que dan como resultado la adquisición de territorios y la distribución espacial en la naturaleza, así como los mecanismos que la subyacen. La Figura 3 resume las evidencias provenientes de estudios en la naturaleza y el laboratorio, y confirma la trascendencia de $G$. omarorum como modelo de elección para el estudio de la territorialidad no reproductiva, en el que es posible postular hipótesis a partir de evidencia conductual, plantear predicciones que pueden ser abordadas en el ambiente natural y también refrendarlas a través del tránsito inverso hacia el laboratorio.

\section{AGRADECIMIENTOS}

Este Ensayo constituye la introducción y antecedentes reformulados así como un breve resumen de los resultados de la tesis de Doctorado PEDECIBABiología de Lucía Zubizarreta, orientada por Ana Silva y co-orientada por Laura Quintana. El desarrollo de la tesis fue posible gracias a: Beca de Doctorado ANII, Beca de finalización CAP, PEDECIBA alícuota para estudiantes, PEDECIBA apoyo para congresos en el exterior, PEDECIBA apoyo para pasantía en la Universidad de San Pablo, Society for Behavioral Neuroendocrinology travel Award para congreso, CSIC MIA para pasantía en University of British Columbia, ELAP de Canadá para pasantía en University of British Columbia. La parte experimental de la tesis contó con la siguiente financiación: FCE _6180; FCE_104272; FCE_136381.

\section{BIBLIOGRAFÍA}

Adams, E. S. (2001). Approaches to the Study of Territory Size and Shape. Annual Review of Ecology and Systematics, 32(1), 277-303. https://doi.org/10.1146/annurev.ecolsys.32.081 501.114034

Adkins-Regan, E. (2005). Hormones and Animal Social Behavior. Princeton University Press.

Armitage, K.B. (1977). Social variety in the yellowbellied marmot: A population-behavioural system. Animal Behaviour, 25, 585-593. https://doi.org/10.1016/0003-3472(77)90108-7

Batista, G., Zubizarreta, L., Perrone, R. \& Silva, A. (2012). Non-sex-biased dominance in a sexually monomorphic electric fish: Fight structure and submissive electric signalling. Ethology, 118(4), 398-410. https://doi.org/ 10.1111/j.1439-0310.2012.02022.x

Borg, B. (1994). Androgens in teleost fishes. Comparative Biochemistry and Physiology Part C: Pharmacology, Toxicology and Endocrinology, 109(3), 219-245.

Briffa, M. \& Sneddon, L. (2010). Contest behavior. Evolutionary behavioral ecology, $246 \mathrm{e} 265$.

Brown, J.L. (1964). The Evolution of Diversity in Avian Territorial Systems. The Wilson Bulletin, 76(2), 160-169. JSTOR.

Brown, J.L. (1975). The evolution of behavior. New York. W. W. Norton. 
Brown, J.L. \& Orians, G. H. (1970). Spacing Patterns in Mobile Animals. Annual Review of Ecology and Systematics, 1, 239-262. JSTOR. https://doi.org/10.1146/annurev.es.01.110170. 001323

Butchart, S.H.M., Seddon, N. \& Ekstrom, J.M.M. (1999). Polyandry and competition for territories in bronze-winged jacanas. Journal of Animal Ecology, 68(5), 928-939. https://doi.org/ 10.1046/j.1365-2656.1999.00341.x

Caldwell, G., Glickman, S. \& Smith, E. (1984). Seasonal aggression independent of seasonal testosterone in wood rats. Proceedings of the National Academy of Sciences of the United States of America, 81(16), 5255-5257. PubMed. https://doi.org/10.1073/pnas.81.16.5255

Chiver, I., Stutchbury, B.J.M. \& Morton, E.S. (2014). Seasonal variation in male testosterone levels in a tropical bird with year-round territoriality. Journal of Field Ornithology, 85(1), 1-9. https://doi.org/10.1111/jofo.12044

Clarke, T.A. (1970). Territorial Behavior and Population Dynamics of a Pomacentrid Fish, the Garibaldi, Hypsypops rubicunda. Ecological Monographs, 40(2), 189-212. https://doi.org/10.2307/ 1942295

Clipperton Allen, A.E., Cragg, C.L., Wood, A.J., Pfaff, D.W. \& Choleris, E. (2010). Agonistic behavior in males and females: Effects of an estrogen receptor beta agonist in gonadectomized and $\mathrm{g} \circ \mathrm{n}$ a d a I l y i n t a c t m i c e. Psychoneuroendocrinology, 35(7), 1008-1022. https://doi.org/10.1016/j.psyneuen.2010.01.002

Clipperton-Allen, A.E., Almey, A., Melichercik, A., Allen, C.P. \& Choleris, E. (2011). Effects of an estrogen receptor alpha agonist on agonistic behaviour in intact and gonadectomized male and female mice. Psychoneuroendocrinology, 36(7), 981995 . ht tps://doi . org/10.1016/ j.psyneuen.2010.12.010

Cunningham, R.L., Lumia, A.R. \& McGinnis, M.Y. (2012). Androgen Receptors, Sex Behavior, and Aggression. Neuroendocrinology, 96(2), 131140. https://doi.org/10.1159/000337663

Davies, N.B. (1976). Food, Flocking and Territorial Behaviour of the Pied Wagtail (Motacilla alba yarrellii Gould) in Winter. Journal of Animal Ecology, 45(1), 235-253. JSTOR. https://doi.org/10.2307/3777

Davies, N.B. \& Hartley, I.R. (1996). Food Patchiness, Territory Overlap and Social Systems: An Experiment with Dunnocks Prunella modularis. Journal of Animal Ecology, 65(6), 837-846. JSTOR. https://doi.org/10.2307/5681

Davies, N.B., Krebs, J. \& West, S. (2012). An introduction to behavioural ecology (4.: ed.). Wiley Blackwell.

Demas, G.E., Cooper, M.A., Albers, H.E. \& Soma, K.K. (2007). Novel Mechanisms Underlying
Neuroendocrine Regulation of Aggression: A Synthesis of Rodent, Avian, and Primate Studies. En A. Lajtha \& J. D. Blaustein (Eds.), Handbook of Neurochemistry and Molecular Neurobiology (pp. 337-372). Springer US. https://doi.org/10.1007/978-0-387-30405-2_8

Dunham, A. (2008). Battle of the sexes: Cost asymmetry explains female dominance in lemurs. Animal Behaviour, 1435-1439. https://doi.org/doi:10.1016/j.anbehav.2008.06. 018

Duque-Wilckens, N. \& Trainor, B.C. (2017). Behavioral Neuroendocrinology of Female Aggression. Oxford Un i versity Press. https://oxfordre.com/neuroscience/view/10.109 3/acrefore/9780190264086.001.0001/acrefore $-9780190264086-e-11$

Eason, P.K., Cobbs, G.A. \& Trinca, K.G. (1999). The use of landmarks to define territorial boundaries. Animal Behaviour, 58(1), 85-91. https://doi.org/10.1006/anbe.1999.1133

Eastman, G., Valiño, G., Radío, S., Young, R.L., Quintana, L., Zakon, H.H., Hofmann, H.A., Sotelo-Silveira, J. \& Silva, A. (2020). Brain transcriptomics of agonistic behaviour in the weakly electric fish Gymnotus omarorum, a wild teleost model of non-breeding aggression. Scientific Reports, 10(1), 9496. https://doi.org/10.1038/s41598-020-66494-9

Elliott, J.M. (1990). Mechanisms Responsible for Population Regulation in Young Migratory Trout, Salmo trutta. III. The Role of Territorial Behaviour. Journal of Animal Ecology, 59(3), 803-818. JSTOR. https://doi.org/10.2307/5015

Fleming, A.S., Phillips, A., Rydall, A. \& Levesque, L. (1988). Effects of photoperiod, the pineal gland and the gonads on agonistic behavior in female golden hamsters (Mesocricetus auratus). Physiology \& Behavior, 44(2), 227-234. https://doi.org/10.1016/0031-9384(88)90143-6

Fowler, C.D., Freeman, M.E. \& Wang, Z. (2003). Newly proliferated cells in the adult male amygdala are affected by gonadal steroid hormones. Journal of neurobiology, 57(3), 257-269.

Fuxjager, M.J., Trainor, B.C. \& Marler, C.A. (2017). What can animal research tell us about the link between androgens and social competition in humans? Hormones and Human Competition, 92, 182-189. https://doi.org/10.1016/ j.yhbeh.2016.11.014

Gill, S.A., Alfson, E.D. \& Hau, M. (2007). Context matters: Female aggression and testosterone in a year-round territorial neotropical songbird (Thryothorus leucotis). Proceedings of the Royal Society B: Biological Sciences, 274(1622), 2187-2194. https://doi.org/ $10.1098 /$ rspb.2007.0457

Grant, J.W.A. (1997). Territoriality. Behavioural ecology of teleost fishes. Oxford: Oxford 
University Press.

Gwinner, E., Rödl, T. \& Schwabl, H. (1994). Pair Territoriality of Wintering Stonechats: Behaviour, Function and Hormones. Behavioral Ecology and Sociobiology, 34(5), 321-327. JSTOR.

Harding, C. (1992). Hormonal modulation of neurotransmitter function and behavior in male songbirds. Poultry science reviews, 4(4), 261273.

Hardy, I.C.W. \& Briffa, M. (2013). Animal Contests. Cambridge University Press. https:// books.google.com.uy/books?id=aQICAQAAQ BAJ

Hau, M., Stoddard, S.T. \& Soma, K.K. (2004). Territorial aggression and hormones during the nonbreeding season in a tropical bird. Hormones and Behavior, 45(1), 40-49. https://doi.org/ 10.1016/j.yhbeh.2003.08.002

Heike Pröhl. (2005). Territorial Behavior in Dendrobatid Frogs. Journal of Herpetology, 39(3), 354-365. https://doi.org/10.1670/162-04A.1

Heimovics, S.A., Ferris, J.K. \& Soma, K.K. (2015). Non-invasive administration of 17â-estradiol rapidly increases aggressive behavior in nonbreeding, but not breeding, male song sparrows. Hormones and Behavior, 69, 31-38. https://doi.org/10.1016/j.yhbeh.2014.11.012

Heimovics, S.A., Trainor, B.C. \& Soma, K.K. (2015). Rapid Effects of Estradiol on Aggression in Birds and Mice: The Fast and the Furious. Integrative and Comparative Biology, 55(2), 281-293. https://doi.org/10.1093/icb/icv048

Hirschenhauser, K. \& Oliveira, R.F. (2006). Social modulation of androgens in male vertebrates: Meta-analyses of the challenge hypothesis. Animal Behaviour, 71(2), 265-277. https://doi.org/10.1016/j.anbehav.2005.04.014

Huang, W.-S., Greene, H.W., Chang, T.-J. \& Shine, R. (2011). Territorial behavior in Taiwanese k u k risnakes (< e m > O ligodon formosanus $</$ em $>$ ). Proceedings of the National Academy of Sciences, 108(18), 7455. https://doi.org/10.1073/pnas.1101804108

Huntingford, F.A. \& Turner, A.K. (1987). The consequences of animal conflict. En F. A. Huntingford \& A. K. Turner (Eds.), Animal Conflict (pp. 227-250). Springer Netherlands. https://doi.org/10.1007/978-94-009-3145-9_9

Hurd, P.L. (2006). Resource holding potential, subjective resource value, and game theoretical models of aggressiveness signalling. Journal of Theoretical Biology, 241(3), 639-648. https://doi.org/10.1016/j.jtbi.2006.01.001

Jalabert, C., Munley, K.M., Demas, G.E. \& Soma, K.K. (2018). Aggressive behavior.

Jalabert, C., Quintana, L., Pessina, P. \& Silva, A. (2015). Extra-gonadal steroids modulate non- breeding territorial aggression in weakly electric fish. Hormones and Behavior, 72, 60-67. https://doi.org/10.1016/j.yhbeh.2015.05.003

Jasnow, A.M., Huhman, K.L., Bartness, T.J. \& Demas, G.E. (2000). Short-Day Increases in Aggression Are Inversely Related to Circulating Testosterone Concentrations in Male Siberian Hamsters (Phodopus sungorus). Hormones and Behavior, 38(2), 102-110. https://doi.org/ 10.1006/hbeh.2000.1604

Keeley, E.R. (2000). An experimental analysis of territory size in juvenile steelhead trout. Animal Behaviour, 59(3), 477-490. https://doi.org/ 10.1006/anbe.1999.1288

King, J.A. (1973). The Ecology of Aggressive Behavior. Annual Review of Ecology and Systematics, 4, 117-138. JSTOR.

Laredo, S.A., Villalon Landeros, R., Dooley, J.C., Steinman, M.Q., Orr, V., Silva, A.L., Crean, K.K., Robles, C.F. \& Trainor, B. C. (2013). Nongenomic effects of estradiol on aggression under short day photoperiods. Hormones and Behavior, 64(3), 557-565. https://doi.org/ 10.1016/j.yhbeh.2013.06.002

Logan, C.A. \& Wingfield, J.C. (1990). Autumnal territorial aggression is independent of plasma testosterone in mockingbirds. Hormones and Behavior, 24(4), 568-581. https://doi.org/ 10.1016/0018-506X(90)90042-V

Maher, C.M. \& Lott, D. F. (2000). A Review of Ecological Determinants of Territoriality within Vertebrate Species. The American Midland Naturalist, 143(1), 1-29. https://doi.org/10.1674/00030031(2000)143[0001:AROEDO]2.0.CO;2

Mayer, A.D. \& Rosenblatt, J.S. (1987). Hormonal factors influence the onset of maternal aggression in laboratory rats. Hormones and behavior, 21(2), 253-267.

McEwen, B.S. \& Wingfield, J.C. (2003). The concept of allostasis in biology and biomedicine. Hormones and Behavior, 43(1), 2-15. https:// doi.org/10.1016/S0018-506X(02)00024-7

Merritt, J.R., Davis, M.T., Jalabert, C., Libecap, T.J., Williams, D.R., Soma, K.K. \& Maney, D.L. (2018). Rapid effects of estradiol on aggression depend on genotype in a species with an estrogen receptor polymorphism. Hormones and behavior, 98, 210-218. https://doi.org/ 10.1016/j.yhbeh.2017.11.014

Metcalfe, N.B., Van Leeuwen, T.E. \& Killen, S. S. (2016). Does individual variation in metabolic phenotype predict fish behaviour and performance? Journal of Fish Biology, 88(1), 298-321. https://doi.org/10.1111/jfb.12699

Micevych, P.E., Hammer, R.P. \& Hammer Jr,R.P. (2005). Neurobiological effects of sex steroid hormones. Cambridge University Press.

Moorcroft, P.R., Lewis, M.A. \& Crabtree, R.L. (2006). 
Mechanistic home range models capture spatial patterns and dynamics of coyote territories in Yellowstone. Proceedings of the Royal Society B: Biological Sciences, 273(1594), 1651-1659. https://doi.org/10.1098/rspb.2005.3439

Moore, M.C. \& Marler, C.A. (1987). Effects of testosterone manipulations on nonbreeding season territorial aggression in free-living male lizards, Sceloporus jarrovi. General and Comparative Endocrinology, 65(2), 225-232. https://doi.org/10.1016/0016-6480(87)90170-5

Morales, J.M., Moorcroft, P.R., Matthiopoulos, J., Frair, J.L., Kie, J.G., Powell, R.A., Merrill, E.H. \& Haydon, D.T. (2010). Building the bridge between animal movement and population dynamics. Philosophical Transactions of the Royal Society B: Biological Sciences, 365(1550), 2289-2301. https://doi.org/10.1098/ rstb.2010.0082

Nelson, R.J. (2006). Biology of Aggression. Oxford University Press, USA.

Nolen, Z.J., Allen, P.E. \& Miller, C. W. (2017). Seasonal resource value and male size influence male aggressive interactions in the leaf footed cactus bug, Narnia femorata. Behavioural Processes, 138, 1-6. https://doi.org/10.1016/ j.beproc.2017.01.020

Perrone, R., Pedraja, F., Valiño, G., Tassino, B. \& Silva, A. (2019). Non-breeding territoriality and the effect of territory size on aggression in the weakly electric fish, Gymnotus omarorum. Acta Ethologica, 22(2), 79-89. https://doi.org/ $10.1007 / \mathrm{s} 10211-019-00309-7$

Perrone, R. \& Silva, A.C. (2018). Status-Dependent Vasotocin Modulation of Dominance and Subordination in the Weakly Electric Fish Gymnotus omarorum. Frontiers in Behavioral Neuroscience, 12, 1. https://doi.org/10.3389/ fnbeh.2018.00001

Piper, W.H., Mager, J.N., Walcott, C., Furey, L., Banfield, N., Reinke, A., Spilker, F. \& Flory, J. A. (2015). Territory settlement in common loons: No footholds but age and assessment are important. Animal Behaviour, 104, 155-163. https://doi.org/10.1016/j.anbehav.2015.03.013

Pradhan, D.S., Newman, A.E., Wacker, D.W., Wingfield, J.C., Schlinger, B.A. \& Soma, K.K. (2010). Aggressive interactions rapidly increase androgen synthesis in the brain during the nonbreeding season. Hormones and behavior, 57(4-5), 381-389.

Quintana, L., Zubizarreta, L., Jalabert, C., Batista, G., Perrone, R. \& Silva, A. (2016). Building the case for a novel teleost model of non-breeding aggression and its neuroendocrine control. 110(3, Part B), 224-232. https://doi.org/ 10.1016/j.jphysparis.2016.11.009

Remage-Healey, L. (2014). Frank Beach Award Winner: Steroids as neuromodulators of brain circuits and behavior. Hormones and behavior, 66(3), 552-560.

Rendon, N.M., Amez, A.C., Proffitt, M.R., Bauserman, E.R. \& Demas, G.E. (2017). Aggressive behaviours track transitions in seasonal phenotypes of female Siberian hamsters. Functional Ecology, 31(5), 1071-1081. https://doi.org/10.1111/1365-2435.12816

Renn, S.C.P., Fraser, E.J., Aubin-Horth, N., Trainor, B.C. \& Hofmann, H.A. (2012). Females of an African cichlid fish display male-typical social dominance behavior and elevated androgens in the absence of males. Hormones and Behavior, 61(4), 496-503. https://doi.org/10.1016/ j.yhbeh.2012.01.006

Richer-de-Forges, M.M., Crampton, W.G.R. \& Albert, J.S. (2009). A New Species of Gymnotus (Gymnotiformes, Gymnotidae) from Uruguay: Description of a Model Species in Neurophysiological Research. Copeia, 2009(3), 538-544. JSTOR.

Rosvall, K.A., Bergeon Burns, C.M., Barske, J., Goodson, J.L., Schlinger, B.A., Sengelaub, D.R. \& Ketterson, E. D. (2012). Neural sensitivity to sex steroids predicts individual differences in aggression: Implications for behavioural evolution. Proceedings of the Royal Society B: Biological Sciences, 279(1742), 3547-3555. https://doi.org/10.1098/rspb.2012.0442

Rosvall, Kimberly A. (2011). Intrasexual competition in females: Evidence for sexual selection? Behavioral Ecology, 22(6), 1131-1140. https://doi.org/10.1093/beheco/arr106

Rosvall, Kimberly A. (2013). Proximate perspectives on the evolution of female aggression: Good for the gander, good for the goose? Philosophical Transactions of the Royal Society B: Biological Sciences, 368(1631), 20130083. https://doi.org/10.1098/rstb.2013.0083

Rosvall, Kimberly A., Bentz, A.B. \& George, E.M. (2019). How research on female vertebrates contributes to an expanded challenge hypothesis. Hormones and Behavior, 104565. https://doi.org/10.1016/j.yhbeh.2019.104565

Rubenstein, D.R. \& Alcock, J. (2018). Animal Behavior (11. ed.). Oxford University Press.

Rubenstein, D.R. \& Wikelski, M. (2005). Steroid hormones and aggression in female Galápagos marine iguanas. Hormones and Behavior, 48(3), 329-341. https://doi.org/10.1016/ j.yhbeh.2005.04.006

Scaia, M.F., Morandini, L., Noguera, C., Trudeau, V.L., Somoza, G.M. \& Pandolfi, M. (2018). Can estrogens be considered as key elements of the challenge hypothesis? The case of intrasexual aggression in a cichlid fish. Physiology \& Behavior, 194, 481-490. https://doi.org/ 10.1016/j.physbeh.2018.06.028

Schlinger, B.A. \& Callard, G.V. (1990). Aromatization 
mediates aggressive behavior in quail. General and Comparative Endocrinology, 79(1), 39-53. https://doi.org/10.1016/0016-6480(90)90086-2

Scotti, M.-A.L., Place, N.J. \& Demas, G.E. (2007). Short-day increases in aggression are independent of circulating gonadal steroids in female Siberian hamsters (Phodopus sungorus). Hormones and Behavior, 52(2), 183 - 190 .https://doi.org/10.1016/ j.yhbeh.2007.03.029

Silva, A.C., Perrone, R., Zubizarreta, L., Batista, G. \& Stoddard, P. K. (2013). Neuromodulation of the agonistic behavior in two species of weakly electric fish that display different types of aggression. The Journal of Experimental Biology, 216(13), 2412. https://doi.org/10.1242/ jeb.082180

Silva, A.C., Zubizarreta, L. \& Quintana, L. (2020). A Teleost Fish Model to Understand Hormonal Mechanisms of Non-breeding Territorial Behavior. Frontiers in Endocrinology, 11, 468. https://doi.org/10.3389/fendo.2020.00468

Smiley, K.O., Ladyman, S.R., Gustafson, P., Grattan, D.R. \& Brown, R.S. (2019). Neuroendocrinology and Adaptive Physiology of Maternal Care. Neuroendocrine Regulation of Behavior, 161210.

Smith, D.C. (1985). Home range and territory in the striped plateau lizard (Sceloporus virgatus). Animal Behaviour, 33(2), 417-427. https:// doi.org/10.1016/S0003-3472(85)80066-X

Smith, J.M. \& Parker, G.A. (1976). The logic of asymmetric contests. Animal Behaviour, 24(1), 159-175. https://doi.org/10.1016/S00033472(76)80110-8

Soma, K. (2006). Testosterone and aggression: Berthold, birds and beyond. Journal of neuroendocrinology, 18(7), 543-551.

Soma, K.K., Tramontin, A.D.,\& Wingfield, J.C. (2000). Oestrogen regulates male aggression in the non-breeding season. Proceedings of the Royal Society of London. Series B: Biological Sciences, 267(1448), 1089-1096. https://doi.org/ 10.1098/rspb.2000.1113

Soma, Kiran K., Rendon, N.M., Boonstra, R., Albers, H.E. \& Demas, G.E. (2015). DHEA effects on brain and behavior: Insights from comparative studies of aggression. DHEA (dehydroepiandrosterone) and sex steroid formation (intracrinology), 145, 261-272. https://doi.org/10.1016/j.jsbmb.2014.05.011

Soma, Kiran K., Scotti, M.-A.L., Newman, A.E.M., Charlier, T.D. \& Demas, G.E. (2008). Novel mechanisms for neuroendocrine regulation of aggression. Frontiers in Neuroendocrinology, 29(4), 476-489. https://doi.org/10.1016/ j.yfrne.2007.12.003

Soma, Kiran K, Sullivan, K.A., Tramontin, A.D., Saldanha, C.J., Schlinger, B.A. \& Wingfield, J.C.
(2000). Acute and chronic effects of an aromatase inhibitor on territorial aggression in breeding and nonbreeding male song sparrows. Journal of Comparative Physiology A, 186(7-8), 759-769.

Soma, Kiran K., Sullivan, K. \& Wingfield, J. (1999). Combined Aromatase Inhibitor and Antiandrogen Treatment Decreases Territorial Aggression in a Wild Songbird during the Nonbreeding Season. General and Comparative Endocrinology, 115(3), 442-453. https://doi.org/10.1006/gcen.1999.7334

Thomas, C.D. \& Kunin, W.E. (1999). The spatial structure of populations. Journal of Animal Ecology, 68(4), 647-657. https://doi.org/ 10.1046/j.1365-2656.1999.00330.x

Trainor, B.C., Kyomen, H.H. \& Marler, C.A. (2006). Estrogenic encounters: How interactions between aromatase and the environment modulate aggression. Frontiers in neuroendocrinology, 27(2), 170-179. https://doi.org/10.1016/j.yfrne.2005.11.001

Trainor, B.C., Lin, S., Finy ${ }^{*}$, M.S., Rowland, M.R. \& Nelson, R. J. (2007). Photoperiod reverses the effects of estrogens on male aggression via genomic and nongenomic pathways. Proceedings of the National Academy of Sciences, 104(23), 9840. https://doi.org/ 10.1073/pnas.0701819104

Trainor, B.C., Sima Finy, M. \& Nelson, R.J. (2008). Rapid effects of estradiol on male aggression depend on photoperiod in reproductively nonresponsive mice. Hormones and Behavior, 53(1), 192-199. https://doi.org/10.1016/ j.yhbeh.2007.09.016

Tsutsui, K., Ukena, K., Usui, M., Sakamoto, H. \& Takase, M. (2000). Novel brain function: Biosynthesis and actions of neurosteroids in neurons. Neuroscience research, 36(4), 261273.

Tsutsui, K. \& Yamazaki, T. (1995). Avian neurosteroids. I. Pregnenolone biosynthesis in the quail brain. Brain Research, 678(1), 1-9. https://doi.org/10.1016/0006-8993(95)00116-8

Vullioud, P., Bshary, R. \& Ros, A.F.H. (2013). Intra- and interspecific aggression do not modulate androgen levels in dusky gregories, yet male aggression is reduced by an androgen blocker. Hormones and Behavior, 64(3), 430-438. https://doi.org/10.1016/j.yhbeh.2013.06.007

Wauters, L. \& Dhondt, A. A. (1992). Spacing behaviour of red squirrels, Sciurus vulgaris: Variation between habitats and the sexes. Animal Behaviour, 43(2), 297-311. https://doi.org/ 10.1016/S0003-3472(05)80225-8

Wingfield, J.C., Lynn, S., \& Soma, K. K. (2001). Avoiding the 'Costs' of Testosterone: Ecological Bases of Hormone-Behavior Interactions. Brain, Behavior and Evolution, 57(5), 239-251. 
https://doi.org/10.1159/000047243

Wingfield, John C., \& Hahn, T. P. (1994). Testosterone and territorial behaviour in sedentary and migratory sparrows. Animal Behaviour, 47(1), 77-89. https://doi.org/10.1006/anbe.1994.1009

Wingfield, John C., \& Monk, D. (1994). Behavioral and Hormonal Responses of Male Song Sparrows to Estradiol-Treated Females during the Nonbreeding Season. Hormones and Behavior, 28(2), 146-154. https://doi.org/10.1006/ hbeh.1994.1012

Woodley, S.K. \& Moore, M.C. (1999). Ovarian Hormones Influence Territorial Aggression in Free-Living Female Mountain Spiny Lizards. Hormones and Behavior, 35(3), 205-214. https://doi.org/10.1006/hbeh.1999.1514

Zubizarreta, L. (2020). Bases hormonales de la agresión territorial no reproductiva. PEDECIBA, UdelaR.

Zubizarreta, L., Quintana, L., Hernández, D., Teixeira de Mello, F., Meerhoff, M., Massaaki Honji, R., Guimarães Moreira, R. \& Silva, A. (2020). Seasonal and social factors associated with spacing in a wild territorial electric fish. PLOS ONE, 15(6), e0228976. https://doi.org/10.1371/ journal.pone.0228976

Zubizarreta, L., Silva, A.C. \& Quintana, L. (2020). The estrogenic pathway modulates non-breeding female aggression in a teleost fish. Physiology \& Behavior, 220, 112883. https://doi.org/10.1016/ j.physbeh.2020.112883

Zubizarreta, L., Stoddard, P.K. \& Silva, A. (2015). Aggression Levels Affect Social Interaction in the Non-Breeding Territorial Aggression of the Weakly Electric Fish, Gymnotus omarorum. Ethology, 121(1), 8-16. https://doi.org/10.1111/ eth.12299

Editor de Sección: Raúl Maneyro 